\title{
Resolution enhancement for lensless mask metrology with RESCAN
}

\author{
Iacopo Mochi, Uldis Locans, Atoosa Dejkameh, Ricarda Nebling, Dimitrios Kazazis, Li-Ting \\ Tseng, and Yasin Ekinci \\ Paul Scherrer Institut, Villigen, Switzerland
}

\begin{abstract}
The EUV photomask is a key component of the lithography process for semiconductor manufacturing. A critical defect on the mask could be replicated on several wafers, causing a significant production yield reduction. For this reason, actinic patterned mask inspection is an important metrology component for EUV lithography. The RESCAN microscope is a lensless imaging platform dedicated to EUV mask defect inspection and metrology. The resolution of the tool is about $35 \mathrm{~nm}$, which is similar to that of state-of-the-art EUV microscopes. To improve the resolution of RESCAN, we designed an upgraded optical layout for the illumination system and we developed a coherent diffraction imaging-compatible method to synthesize a custom pupil structure. This new scheme will enable a lensless EUV microscope with a resolution down to $20 \mathrm{~nm}$ and thereby allow mask review capabilities for future technology nodes with EUV lithography.
\end{abstract}

Keywords: EUV mask review, inspection, defects, lensless imaging, coherent diffraction imaging, ptychography.

\section{INTRODUCTION}

As the semiconductor technology nodes become smaller, the requirements and specifications for extreme ultraviolet (EUV) mask metrology become more and more demanding. To address the future challenges in the EUV lithography infrastructure, at the Paul Scherrer Institute we have been developing RESCAN, a lensless actinic EUV microscopy platform based on coherent diffraction imaging (CDI). RESCAN is capable of operating in die-to-die and in die-to-database mode and can detect phase and amplitude defects as small as $50 \times 50 \mathrm{~nm}^{2}$ (on mask). ${ }^{1}$ RESCAN can also operate through pellicle and can be used to characterize pellicle scattering and to inspect the pellicle surface for defects. ${ }^{2}$

RESCAN is a prototype developed to demonstrate the feasibility of lensless APMI. As such, it has a few limitations that could be easily overcome by upgrading its components, like the mask scanning stage, the vacuum chamber and the detector. The ultimate resolution of the microscope though, depends fundamentally on the numerical aperture (NA) of the system. Considering the reflectance of the standard EUV multilayer stack as a function of the reflection angle, it is clear that high frequency components in the diffraction patterns can get cut, limiting the maximum achievable imaging resolution. Another fundamental limitation of RESCAN, common to other EUV microscopes designed to work in reflection mode with a Chief Ray Angle (CRA) of $6^{\circ}$, is the shadowing of the condenser that limits the lower light collection angle to $-6^{\circ}$. To overcome these limitations, we designed a new optical system and we developed a method to combine multiple diffraction patterns obtained with different incidence angles to synthesize a larger pupil without compromising the spatial coherence of the illumination.

\footnotetext{
Further author information: E-mail: iacopo.mochi@psi.ch
} 


\section{RESCAN PRACTICAL RESOLUTION LIMIT}

The current optical layout of RESCAN is shown in Figure 1. The XIL-II beamline at the Swiss Light Source synchrotron (SLS) produces a beam with a wavelength $\lambda=13.5 \mathrm{~nm}$ and a bandwidth $\lambda / \Delta \lambda=1500$. The EUV beam goes through a circular aperture with a radius $r$ ranging from 0.25 to $2 \mathrm{~mm}$ and reaches a condenser mirror $\left(\mathrm{M}_{1}\right)$ which focuses the beam at a distance of $120 \mathrm{~mm}$. A flat folding mirror $\left(\mathrm{M}_{2}\right)$ directs the beam focus on the surface of the sample with an CRA of $6^{\circ}$. The size of the illumination spot on the sample can be controlled by adjusting the position $\mathrm{M}_{1}$ and it is usually kept between $1.5 \mu \mathrm{m}$ and $5 \mu \mathrm{m}$ radius. The intensity of the far-field diffraction pattern is recorded by a CCD detector lying on a plane perpendicular to the reflected beam. The complex image of the sample is reconstructed from the recorded diffraction data using ptychography. ${ }^{3,4}$

As for conventional optical systems, the resolution limit of a CDI instrument depends on the wavelength and on the NA. In this case, the NA is defined by the angular aperture of the detector. The CCD is positioned at a distance of $d=62 \mathrm{~mm}$ from the sample and its side length is $L=27.6 \mathrm{~mm}$. The maximum NA is therefore:

$$
\mathrm{NA}_{\max }=\sin \left(\arctan \left(\frac{L}{2 d}\right)\right)=0.218
$$

which corresponds to an angular aperture of the detector of $\pm 12.5^{\circ}$. Usually, to increase the resolution, it is sufficient to increase the angular aperture of the detector. Unfortunately, working in reflection mode, the illumination optics $\left(\mathrm{M}_{2}\right)$ shadows a portion of the detector as shown in the diagram in Figure 1. The CRA of $6^{\circ}$, chosen to match the illumination conditions of the EUV scanners used in the lithography process, fixes the maximum value of the collection semi-angle to $12^{\circ}$ on the side of $\mathrm{M}_{2}$.

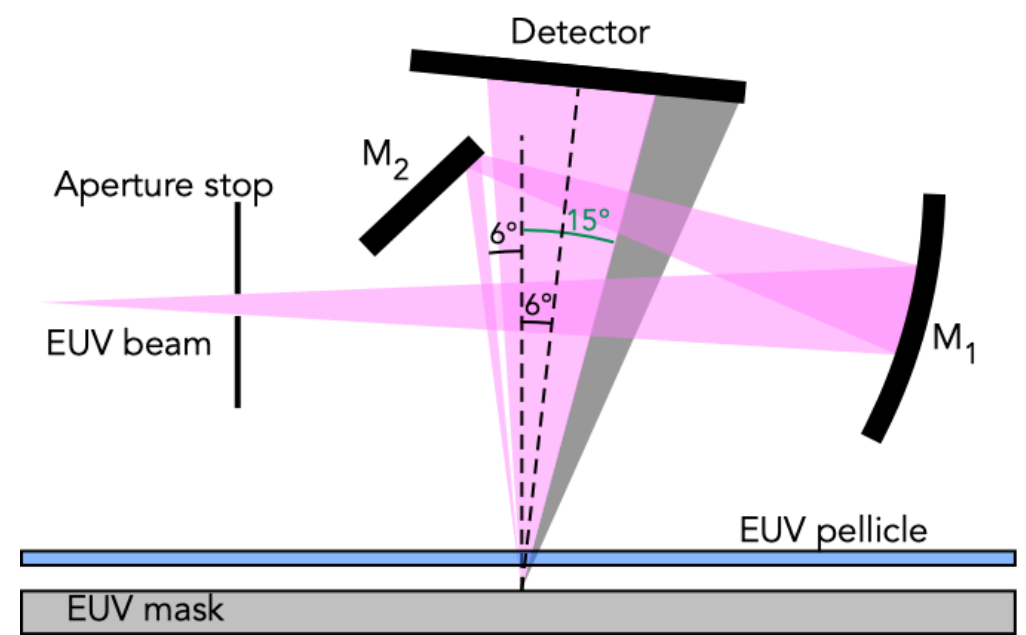

Figure 1. Optical layout of the RESCAN microscope. The illumination system consists of a variable aperture stop, a toroidal condenser mirror $\left(M_{1}\right)$ and a flat folding mirror $M_{2}$ which reflects the focused beam on the sample with an angle of incidence of $6^{\circ}$. The polarization of the EUV beam on the sample is perpendicular to the incidence plane. The diffracted intensity is recorded by a CCD detector at a distance of $62 \mathrm{~mm}$ from the sample.

On the opposite side of the detector, closer to $\mathrm{M}_{1}$ in Figure 1, the collection semi-angle is limited by the reflectance of the mask itself (gray cone). The EUV mask is coated with a Mo-Si multilayer to guarantee a high reflectance at a wavelength of $13.5 \mathrm{~nm}$ in a range of incidence angles centered at $6^{\circ}$. A typical structure for the multilayer is described in Table 1 and its reflectance for an s-polarized beam, calculated using a simple matrix multiplication method ${ }^{6}$ is shown in figure $2 \mathrm{~A}$. A customizable MATLAB ${ }^{\circledR}$ function implementing the method is available online. ${ }^{7}$ Figure $2 \mathrm{~B}$ shows the multilayer reflectance response seen from the detector plane. The center of the detector corresponds to a reflection angle of $6^{\circ}$ and the red and green circles represent a NA of 0.33 and 0.22 respectively. The map shows that for a NA of 0.22 , a crescent-shaped area of the aperture is effectively vignetted by the multilayer reflectance response. This effect can be observed in the diffraction pattern intensity shown in Figure 3B. This diffraction pattern was generated illuminating a sample with a Siemens star pattern. 


\begin{tabular}{c|c|c|c|c} 
Layer & Material & Thickness $[\mathrm{nm}]$ & $\mathrm{n}$ & $\mathrm{k}$ \\
\hline 1 & $\mathrm{Ru}$ & 2 & 0.886358 & -0.01707 \\
2 & $\mathrm{Si}$ & 2.506 & 0.99932 & -0.00183 \\
3 & $\mathrm{MoSi}_{2}$ & 0.802 & 0.9693 & -0.00433 \\
4 & $\mathrm{Mo}$ & 1.904 & 0.92108 & -0.00644 \\
5 & $\mathrm{MoSi}_{2}$ & 1.844 & 0.9693 & -0.00433 \\
6 & $\mathrm{Si}$ & $1 \mathrm{e} 6$ & 0.99932 & -0.00183
\end{tabular}

Table 1. Standard multilayer stack for EUV masks. The multilayer is constituted by $40 \mathrm{Mo} / \mathrm{Si}$ bi-layers. At the interface between the layers, an intermixing region of $\mathrm{MoSi}_{2}$ with variable thickness forms. The multilayer is capped with a 2-nm $\mathrm{Ru}$ layer to improve its resistance to oxidation. ${ }^{5}$

The sample was manufactured depositing a 140-nm-thick HSQ layer on a silicon wafer coated with an EUV multilayer and pattering it with e-beam lithography. Although the structure of the multilayer on the sample is not precisely the one listed in Table 1, the asymmetry of the diffraction pattern is consistent with the vignetting caused by the sample reflectance.

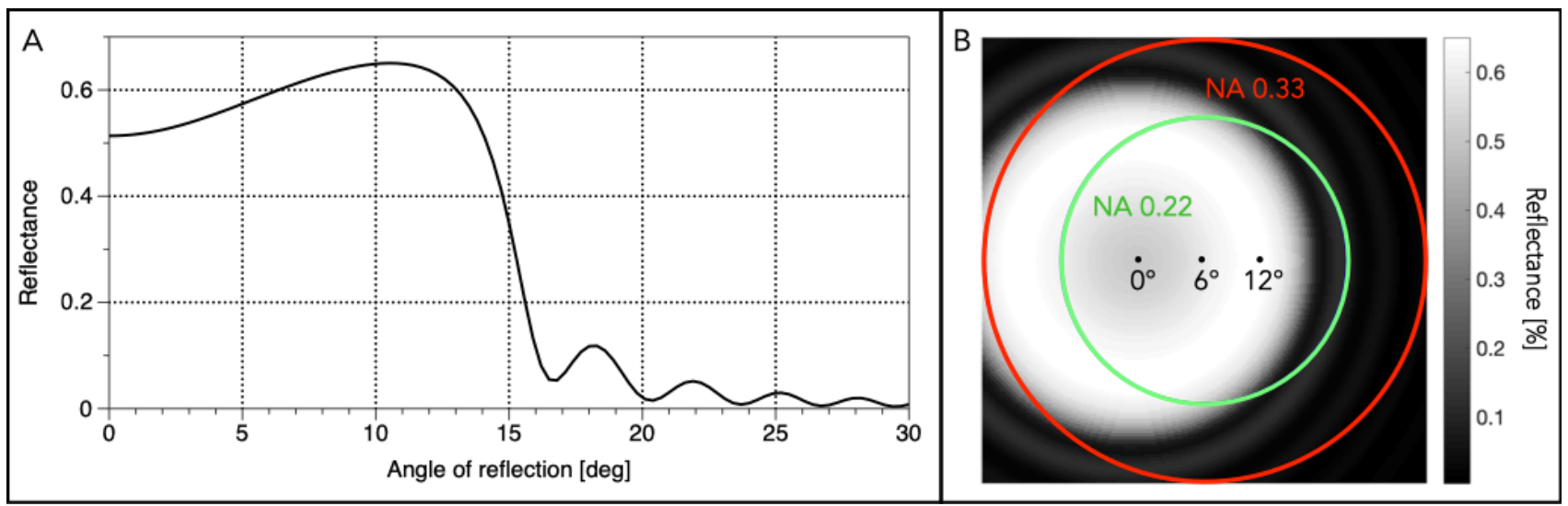

Figure 2. Reflectance of a standard EUV multilayer mirror at a wavelength of $13.5 \mathrm{~nm}$. A. Reflectance curve as a function of the angle of incidence for the multilayer described in Table 1. B. Multilayer reflectance response map seen from the detector plane. The detector plane is centered on a beam reflected at $6^{\circ}$ and it is perpendicular to the reflection vector.

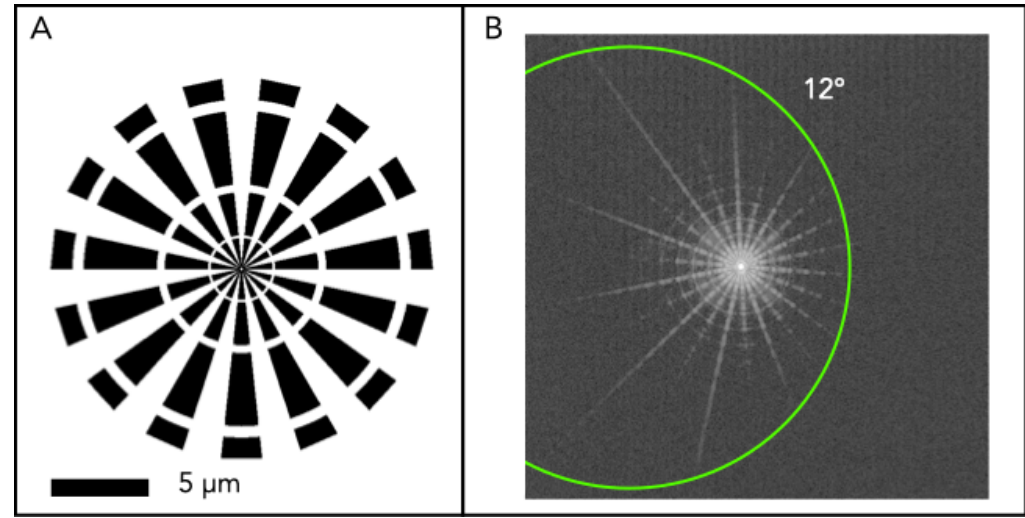

Figure 3. A. Layout detail of an EUV mask sample ${ }^{1}$ with a Siemens star pattern. B. Diffraction pattern recorded with RESCAN illuminating the central part of the Siemens star with an angle of incidence of $6^{\circ}$. The green circle represents diffraction angles of $12^{\circ}$ beyond which value, the multilayer reflectance starts decreasing. This is the reason of the asymmetry of the diffraction pattern in the figure.

Considering both the limits imposed by the shadowing of the illumination optics and by the multilayer reflectance, we calculated the modulation transfer function for different nominal NA values. Figure 4A shows 
the modulation transfer function for horizontal features, parallel to the light incidence plane. In this case, the vignetting from the multilayer is symmetric and the shadowing due to the illumination optics doesn't affect the diffraction, nevertheless the reflectance of the sample limits the useful NA to about 0.3. Figure 4B shows the modulation transfer function for vertical features, perpendicular to the light incidence plane, for which the reflectance limitation effect is more severe and the $\mathrm{M}_{2}$ mirror blocks one side of the diffraction pattern. The plot shows that the MTF values do not change significantly for NA values larger than 0.22 , which is close to the current value used in RESCAN.

\section{A}

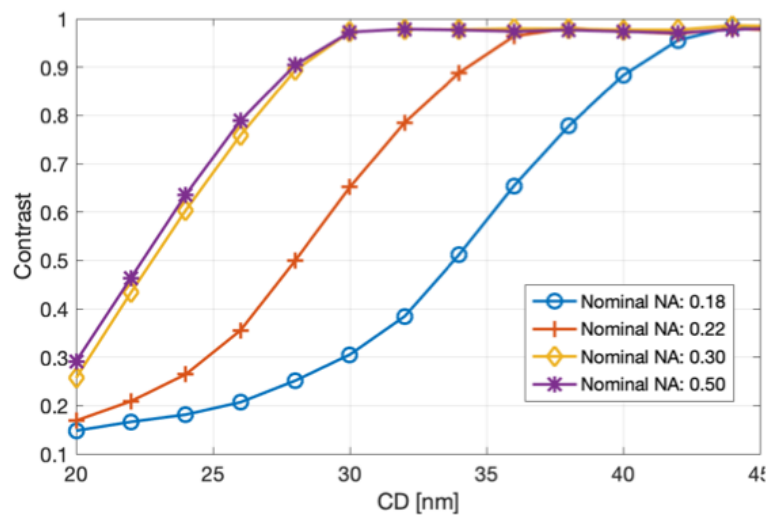

B

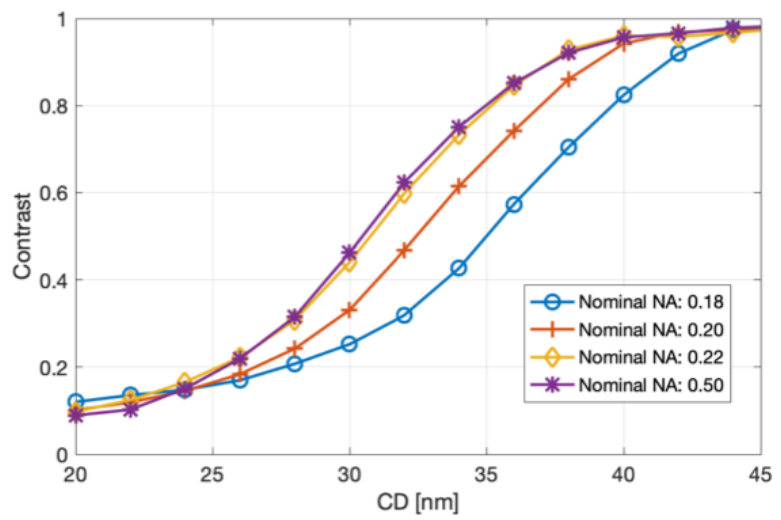

Figure 4. A. Modulation transfer function for EUV mask features perpendicular to the light incidence plane. The angular dependency of the multilayer reflectance limits the effective NA to about 0.3. B. Modulation transfer function for EUV mask features parallel to the light incidence plane. The angular dependency of the multilayer reflectance and the shadow cast by the illumination optics limit the effective NA to about 0.22 .

\section{RESOLUTION ENHANCEMENT}

It is possible to overcome the limitations outlines in Section 2 by synthesizing a large NA combining multiple samplings of the diffraction angular space. This requires an upgrade of the illumination system to allow for variations of the CRA and the development of an image reconstruction algorithm that can combine the diffraction patterns collected with different incidence angles. In the following Section, the optical layout and results showing the proof of the concept are discussed.

\subsection{Illumination system}

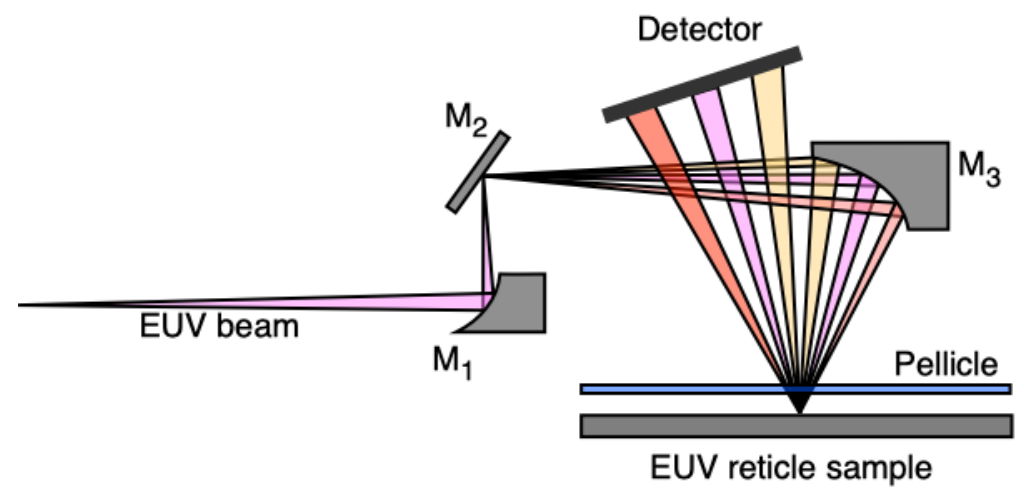

Figure 5. The EUV beam is focused using an off-axis paraboloidal mirror $\left(\mathrm{M}_{1}\right)$ on the surface of a tip-tilt mirror $\left(\mathrm{M}_{2}\right)$. The ellipsoidal condenser mirror $\left(\mathrm{M}_{3}\right)$ re-images on the surface of the sample the focal point on the surface of $\mathrm{M}_{2}$. Changing the angle of $\mathrm{M}_{3}$, we control the angle of incidence of the beam on the sample. 
We designed a Fourier synthesis illuminator ${ }^{8,9}$ to control the incidence angle of the beam on the sample surface. The working concept of the new illuminator is shown in Figure 5. The EUV beam from the synchrotron is focused on the surface of a flat tip-tilt mirror $\left(\mathrm{M}_{2}\right)$. The ellipsoidal condenser mirror $\left(\mathrm{M}_{3}\right)$, re-images the focus of the beam on the surface of the sample. The pivot point of $\mathrm{M}_{2}$ coincides with the incidence point of the incoming beam. By changing the angle of $\mathrm{M}_{2}$ we can control the angle of incidence of the beam without changing its position on the mask. The condenser mirror allows changing the incidence angle between $0^{\circ}$ and $\alpha_{\max }$, the maximum value allowed by the multilayer. Considering the reflectance curve in Figure $2 \mathrm{~A}, \alpha_{\max }$ could be chosen to be around $15^{\circ}$, allowing a maximum (synthesized) NA equal to $\sin 2 \alpha_{\max }=0.5$ and a corresponding resolution below $20 \mathrm{~nm}$.

\subsection{Fourier synthesis image reconstruction}

In conventional microscopy and in lens-based EUV microscopy, it is possible to reconstruct the phase of the sample under investigation and to increase the resolution limit of the microscope by using Fourier ptychography. ${ }^{10,11}$ This method consists in collecting a set of images of the same sample each with a different illumination angle. The illumination angles are chosen in an array with a step size smaller than the the collection angle of the objective optics, in order to guarantee some overlap between the diffraction spectra. Using an algorithm substantially identical to the one employed in conventional ptychography, the images are used to synthesize a larger complex diffraction pattern from which a high-resolution image is calculated.

Currently, to image a mask in RESCAN, we collect a set of diffraction intensity patterns scanning the sample under a fixed-angle EUV illumination probe. The complex amplitude of the mask is then reconstructed using ptychography. The Fourier synthesis illuminator outlined in Section 3.1 will be used to shift the diffraction orders that would normally be cut by the multilayer reflectance response or by the shadow of the condenser optics, in the angular range visible to the detector. To allow the maximum illumination flexibility, the condenser mirror will allow to have up to normal incidence illumination on the sample. This will limit the detector NA to $\sin 6^{\circ}=0.10$ and the corresponding resolution to about $80 \mathrm{~nm}$. To synthesize a large numerical aperture, we can collect several datasets with different illumination angles and reconstruct the corresponding images. Using Fourier ptychography, we can finally reconstruct a high-resolution image corresponding to a numerical aperture synthesized from the range of illumination angles we used.

To test this method before upgrading illumination system, we used RESCAN to collect a diffraction dataset with a detector NA of 0.2 and we simulated a variation in the illumination angle by shifting the diffraction patterns on the detector plane and cropping their central part to a NA value of 0.07 . We simulated an array of $5 \times 5$ illumination conditions with tangential incidence angle $\left(\mathrm{AOI}_{T}\right)$ from $-1.1^{\circ}$ to $13.1^{\circ}$ and sagittal incidence angle $\left(\mathrm{AOI}_{S}\right)$ from $-7.1^{\circ}$ to $7.1^{\circ}$. Three of the specific cases are shown in Figure 6 .

The sample used for this experiment is a programmed defect mask used to test RESCAN's resolution limit. ${ }^{12}$ We reconstructed the a low-resolution image of the mask from each dataset as shown in the examples in Figure 6 and we used Fourier ptychography to synthsize the high resolution image in Figure 7.

In this experiment we did not expect to improve the resolution of RESCAN since we are not actually changing the illumination angle and the diffraction patterns corresponding to higher reflection angles are still partially vignetted (see Figure 6C). It is important to note that in this experiment we assumed that changing the illumination angle will simply shift the diffraction pattern on the detector surface and we neglected the effects of the 3D topography of the EUV mask.

\section{CONCLUSIONS}

The current resolution limit of the RESCAN microscope depends critically on the reflectance of the EUV mask multilayer. We estimated that the current NA of the instrument is already the maximum value possible and increasing the angular acceptance angle of the detector, by choosing a larger one or by moving it closer to the sample, would not improve the resolution. To overcome this limit, we designed a new illumination optics that will allow to change the angle of incidence of the EUV probe on the sample in order to shift the diffraction pattern. We will use the new illuminator to collect the diffraction orders that would otherwise be vignetted by the multilayer reflectance or by the shadow of the illumination optics. 
A
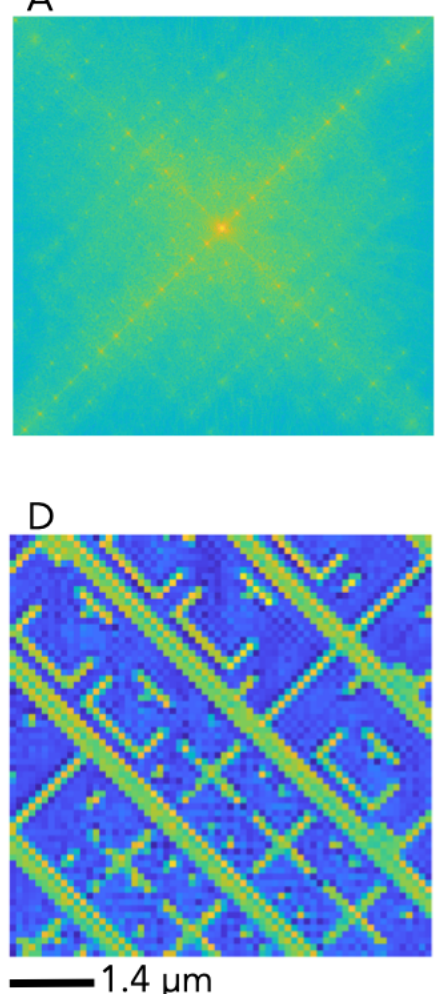

B

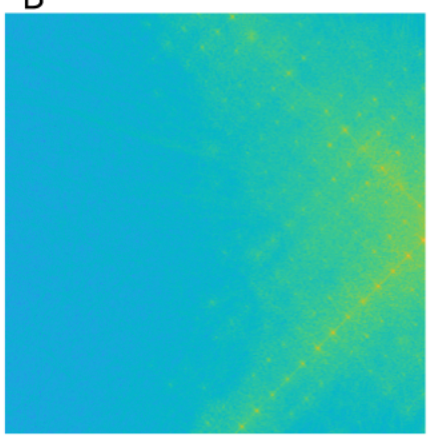

$\mathrm{E}$

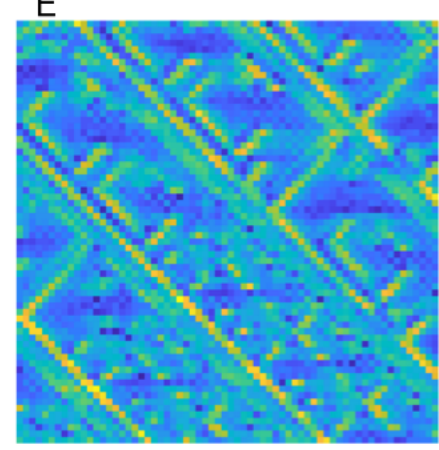

C

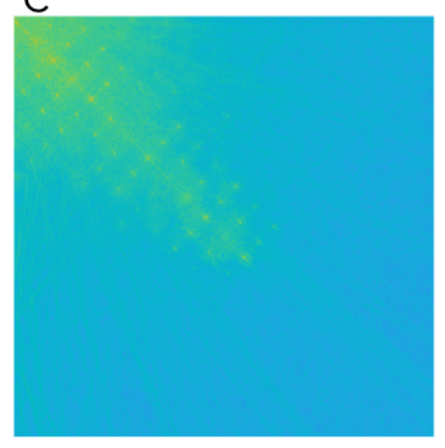

$\mathrm{F}$

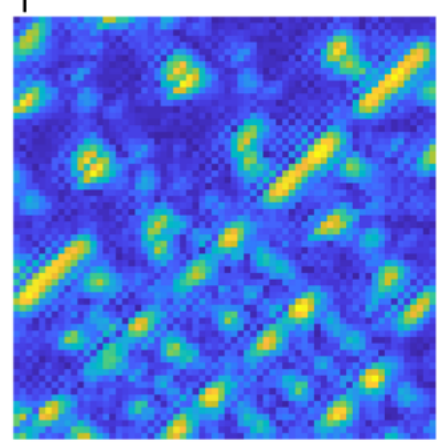

$1.4 \mu \mathrm{m}$

Figure 6. A. Diffraction pattern of the sample corresponding to a tangential angle of incidence $\mathrm{AOI}_{T}=6^{\circ}$ and a sagittal angle of incidence $\mathrm{AOI}_{S}=0$. B. Diffraction pattern of the sample corresponding to $\mathrm{AOI}_{T}=2.4^{\circ}$ and $\mathrm{AOI}_{S}=0$. $\mathbf{C}$. Diffraction pattern of the sample corresponding to $\mathrm{AOI}_{T}=9.6^{\circ}$ and $\mathrm{AOI}_{S}=3.6^{\circ}$. D. Detail of the image reconstructed from A. E. Detail of the image reconstructed from B. F. Detail of the image reconstructed from C.

We proposed a method based on ptychography and Fourier ptychography to combine the diffraction data collected by RESCAN to synthesize a high-resolution complex image of the mask. We successfully tested the method with a diffraction dataset collected with RESCAN, without actually changing the illumination angle, but rather shifting the diffraction patterns and cropping their central part. In this experiment, we neglected the $3 \mathrm{D}$ topography of the mask surface assuming that the diffraction pattern will simply shift when the illumination angle changes. The validity of the method must still be tested on 3D EUV masks, either experimentally or with simulations, although previous Fourier ptychography reconstructions of EUV mask samples have been carried out successfully under the same assumptions. ${ }^{11}$

In addition to increasing the resolution limit of RESCAN, this method will make it possible to synthesize customized illumination pupils, paving the way towards lensless EUV mask review applications.

\section{ACKNOWLEDGMENTS}

The authors wish to thank Michaela Vockenhuber, Markus Kropf and José Gabadinho for their technical support for the measurements carried out at the SLS. This project has received funding from the Electronic Component Systems for European Leadership Joint Undertaking under grant agreement No 783247-TAPES3. This Joint Undertaking receives support from the European Unions Horizon 2020 research and innovation programme and Netherlands, Belgium, France, Germany, Israel. 
A

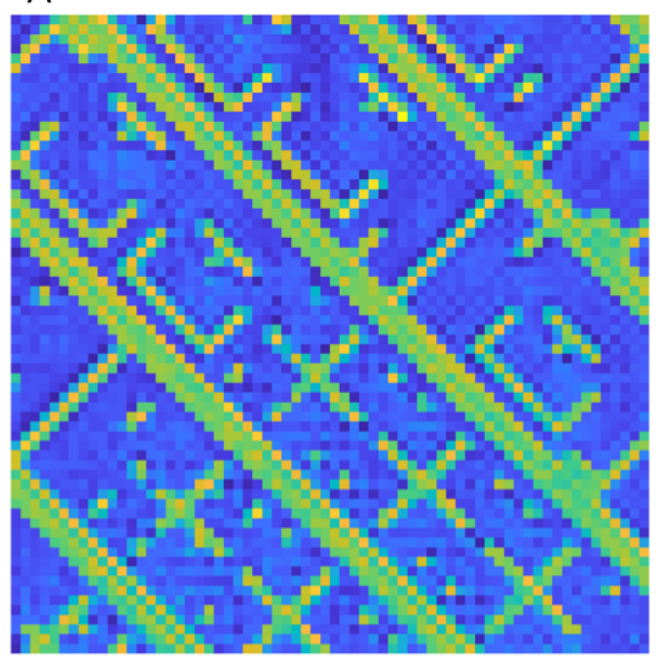

$1.4 \mu \mathrm{m}$
B

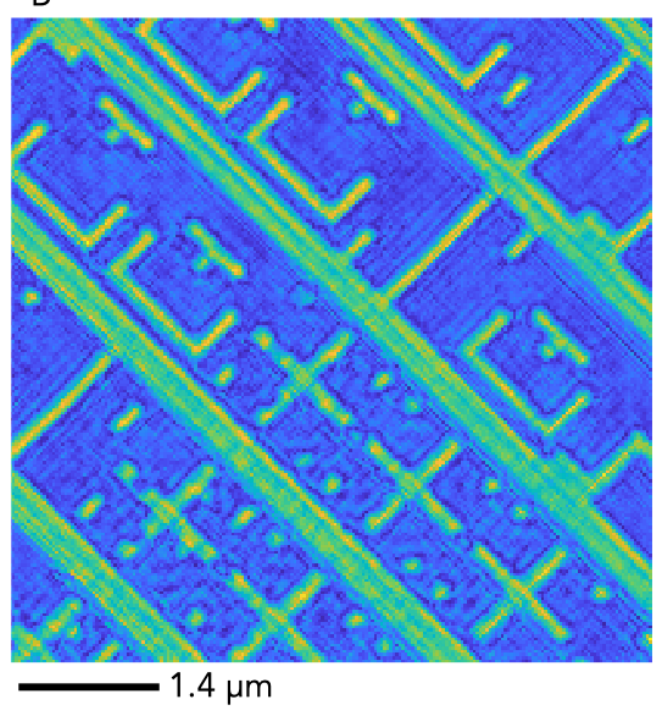

Figure 7. A. Detail of the low-resolution image reconstructed with conventional ptychography from a detector with an $\mathrm{NA}=0.07$. B. Detail of the high-resolution image reconstructed with Fourier ptychography from a set of 25 low-resolution images obtained simulating illumination CRAs from $-1.1^{\circ}$ to $13.1^{\circ}$. The effective value of the synthesized NA is 0.19 .

\section{REFERENCES}

[1] Mochi, I., Fernandez, S., Nebling, R., Locans, U., Helfenstein, P., Rajeev, R., Dejkameh, A., Kazazis, D., Tseng, L.-T., and Ekinci, Y., "Absorber and phase defect inspection on euv reticles using RESCAN," Proc.SPIE 10957 (2019). doi:10.1117/12.2515160.

[2] Mochi, I., Timmermans, M. Y., Gallagher, E. E., Mariano, M., Pollentier, I., Rajendran, R., Helfenstein, P., Fernandez, S., Kazazis, D., and Ekinci, Y., "Experimental evaluation of the impact of carbon nanotube euv pellicles on reticle imaging," Journal of Micro/Nanolithography, MEMS, and MOEMS 18(1), $1-7-7$ (2019). doi:10.1117/1.JMM.18.1.014002.

[3] Helfenstein, P., Rajeev, R., Mochi, I., Kleibert, A., Vaz, C. A. F., and Ekinci, Y., "Beam drift and partial probe coherence effects in EUV reflective-mode coherent diffractive imaging," Opt. Express 26, 12242-12256 (Apr 2018). [doi:10.1364/OE.26.012242].

[4] Thibault, P., Dierolf, M., Bunk, O., Menzel, A., and Pfeiffer, F., "Probe retrieval in ptychographic coherent diffractive imaging," Ultramicroscopy 109(4), 338 - 343 (2009).

[5] Bajt, S., Alameda, J. B., Barbee Jr., T. W., Clift, W. M., Folta, J. A., Kaufmann, B. B., and Spiller, E. A., "Improved reflectance and stability of Mo/Si multilayers," Optical Engineering 41(8), 1797 - 1804 8 (2002).

[6] Hecht, E., [Optics (4th Edition)], 426-428, Addison-Wesley (2001).

[7] Mochi, I., "Multilayer-mirror." https://zenodo.org/badge/latestdoi/207020995 (September 2019).

[8] Goldberg, K. A., Mochi, I., Rekawa, S. B., Smith, N. S., Macdougall, J. B., and Naulleau, P. P., "An euv fresnel zoneplate mask-imaging microscope for lithography generations reaching $8 \mathrm{~nm}$," Proc.SPIE $\mathbf{7 9 6 9}$ (2011).

[9] Naulleau, P. P., Goldberg, K. A., Batson, P., Bokor, J., Denham, P., and Rekawa, S., "Fourier-synthesis custom-coherence illuminator for extreme ultraviolet microfield lithography," Appl. Opt. 42, 820-826 (Feb 2003).

[10] Zheng, G., Horstmeyer, R., and Yang, C., "Wide-field, high-resolution Fourier ptychographic microscopy," Nature Photonics 7, 739-745 (07 2013).

[11] Wojdyla, A., Benk, M. P., Naulleau, P. P., and Goldberg, K. A., "EUV photolithography mask inspection using Fourier ptychography," Proc.SPIE 10656 (2018). doi:10.1117/12.2307860. 
[12] Mochi, I., Kazazis, D., Tseng, L.-T., Fernandez, S., Rajeev, R., Locans, U., Dejkameh, A., Nebling, R., and Yasin, E., "Lensless metrology for semiconductor lithography at EUV," Proc.SPIE 11057 (2019). doi:10.1117/12.2534350.

Proc. of SPIE Vol. 11147 111471D-8 